\title{
Falcon 4 performance validation by single event analysis
}

Jeroen Keizer ${ }^{1}$, Gerald van Hoften ${ }^{2}$, Jaap Mulder ${ }^{1}$ and Gijs van Duinen ${ }^{1}$

${ }^{1}$ Thermo Fisher Scientific, United States, ${ }^{2}$ Thermo Fisher Scientific, Eindhoven, Noord-Brabant, Netherlands

Each Falcon 4 camera that leaves our factory performs according to its committed specifications. Traditionally, integrating TEM cameras (e.g. FEI Eagle and FEI Falcon 2) are characterized by Detective Quantum Efficiency (DQE) [1,2,3]. For electron counting cameras (e.g. Thermo Scientific Falcon 3EC and Falcon 4) a DQE measurement is not trivial; it is time consuming and error prone, even in a standardized manufacturing environment.

DQE is widely accepted as important performance metric for TEM cameras, but the DQE measurement procedure (specifically the MTF measurement) is not valid at dose rates typically used in CryoEM. Since the electron counting process is non-linear, an MTF can only be measured reliably at extremely low dose rates. At dose rates typically used in CryoEM, the MTF and consequently the DQE will be overestimated [3]. In fact, with increasing dose rate, the DQE can be made to appear $>100 \%$, which of course is non physical.

Thermo Fisher Scientific therefore proposes a more reliable verification of performance, based on the analysis of single electron events. Our analysis captures the characteristics of single electron events in a limited number of critical parameters. Determination of these parameters is straightforward and unambiguous. We refer to those as Critical to Quality $(\boldsymbol{C T Q})$ parameters.

The first and most important CTQ parameter for an electron counting camera is the event signal-to-noise ratio (event SNR). The signal generated by a single electron varies and is statistically described by a Landau distribution. The peak of this distribution (Landau peak) is the most likely signal to occur. Our event SNR is defined as the ratio of the Landau peak and the noise RMS value. If the event SNR is too low, the front tail of the Landau distribution overlaps with the end tail of the dark noise distribution, see figure 1 - right. If the event SNR is high, then the two distributions are clearly separated, thus electron events and noise can be clearly distinguished, see Figure 1 - left.

In case event SNR is low, the event detection threshold (TH) cannot be set to an optimal level: a too low $\mathrm{TH}$ will result in false detections which will deteriorate the image quality especially at low dose. A too high TH will remove the false detections, but real electron events (signal) will be missed, reducing DQE. The Falcon 4 sensor was designed with 14um pixels, optimized for a very large event SNR.

The second important CTQ parameter is the event size. The event size is an optimization between coincidence loss and subpixel localization accuracy. The event needs to be slightly larger than a pixel to find the impact location with sub-pixel accuracy, but overlap of nearby events must be minimized. Falcon 4 has been optimized to achieve the lowest coincidence loss while maintaining accurate sub pixel localization.

As stated, executing low dose DQE measurements in the factory is not realistic. Determining the CTQ parameters is the best way to guarantee electron counting camera performance. The CTQ method is not dependent on sample, operator or microscope; this makes it very reliable and the best guarantee that an electron counting camera performs according to its committed specifications. 

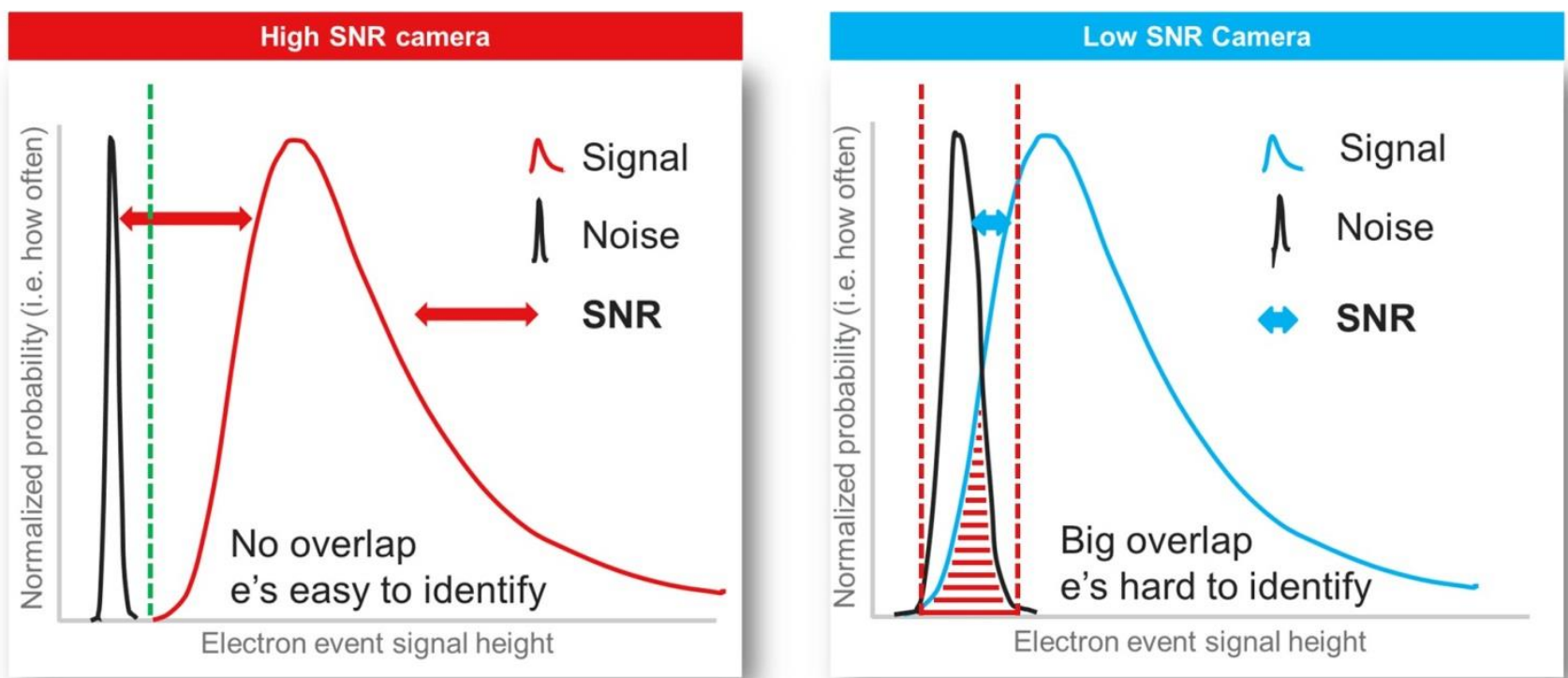

Figure 1. Normalized Noise and Landau distributions. Left: high event SNR. Right: low event SNR.

\section{References}

[1] McMullan G, Chen S, Henderson R, Faruqi AR. Detective quantum efficiency of electron area detectors in electron microscopy. Ultramicroscopy. 2009a; 109:1126-1143.

[2] Faruqi, A.R. \& McMullan, G.. (2017). Direct imaging detectors for electron microscopy. Nuclear Instruments and Methods in Physics Research Section A: Accelerators, Spectrometers, Detectors and Associated Equipment. 878. 10.1016/j.nima.2017.07.037.

[3] Kuijper, Maarten \& Hoften, Gerald \& Janssen, Bart \& Geurink, Rudolf \& De Carlo, Sacha \& Vos, Matthijn \& Duinen, Gijs \& Haeringen, Bart \& Storms, Marc. (2015). FEI's direct electron detector developments: Embarking on a revolution in cryo-TEM. Journal of structural biology. 192. 10.1016/j.jsb.2015.09.014. 